\title{
PHYSIOTHERAPY AND REHABIITATION APPLICATIONS IN LIPEDEMA MANAGEMENT: A LITERATURE REVIEW
}

\author{
M. Esmer, F.J. Schingale, D. Unal, M.V. Yazıc, N.A. Güzel
}

Department of Physiotherapy and Rehabilitation (ME,NAG), Faculty of Health Sciences, Gazi University, Ankara, Turkey; Lympho Opt Fachklinik (FJS), Hohenstadt, Germany; Simav Health Care Vocational School; (DU), Kutahya Health Sciences University, Kütahya, Turkey; Faculty of Health Sciences (MVY), Department of Physiotherapy and Rehabilitation, Yuksek Ihtisas University, Ankara, Turkey

\section{ABSTRACT}

Lipedema is a chronic and progressive disease of adipose tissue caused by abnormal fat accumulation in subcutaneous tissue. Although there is no known cure for lipedema, possible complications can be prevented with conservative and surgical treatments. One of the conservative treatment options is physiotherapy and rehabilitation (PR). When the literature is examined, few studies focusing on the efficacy of PR were found for this patient group. The purpose of this review is to provide a better understanding of the effectiveness of PR applications by compiling existing studies. A bibliographic PubMed search was performed for published studies regarding $P R$ in lipedema management in June 2019 including the last 58 years (1951-2019). Articles were chosen by reading the abstracts and subsequently data were analyzed by reading the entire text through full-text resources. A total of 15 studies met inclusion criteria. Results document how lipedema patients are benefited by $P R$ and the effectiveness of different types of PR programs. The current review also showed that complex decongestive physiotherapy, gait training, hydrotherapy, aerobic exercise, and resistance exercise training each have value in the management of lipedema. The effects of PR for the treatment of lipedema are variable among studies, although overall PR seems to be effective in lipedema management. Although physiotherapy applications have a potentially important role in the management of lipedema, they should be used in combination with other treatment modalities. More studies with higher quality are needed to fully demonstrate the effect and efficacy of PR in lipedema patients.

Keywords: lipedema, literature review, physiotherapy and rehabilitation, complex decongestive therapy, walk training

Lipedema (lipoedema in Europe), first described by Allen and Hines in 1940, is a chronic and progressive disease of adipose tissue caused by abnormal fat accumulation in subcutaneous tissue. $(1,2)$. Although prevalence of lipedema is not known exactly, it has been reported in one study that it affects approximately $11 \%$ of women worldwide and that $80 \%$ of patients have upper limb involvement $(3,4)$. The etiology of lipedema is not fully understood, however many different causes have been proposed. The causes are postulated as either genetic factors or 
hormonal changes. Genetic background with familial predisposition has been described in $60 \%$ of lipedema patients (5-7).

Lipedema is diagnosed based on a patient's medical history and physical examination. To diagnose lipedema correctly, it is essential for it to be clearly distinguished from other diseases that can cause edema or swelling in the lower extremities. Lipedema can be confused with lipohypertrophy in obesity, lymphedema, or phlebedema (8). Understanding the clinical features and the patients' differences in medical histories with lipedema should enable clinicians to make appropriate decisions in planning treatment.

The severity of lipedema is classified according to the surface of the skin and structural changes in the adipose tissue at the extremities. In Stage 1 lipedema, the patient has a normal skin surface with increased underlying fat. In Stage 2, the skin has uneven surface and nodules, and in Stage 3, there are large extrusions of skin and subcutaneous adipose tissue (9).

According to Schingale there are 5 different types of lipedema (10):

- Type I: level of the adipose tissue is increased on buttocks and thighs.

- Type II: lipedema extends to the knees with formation of fat pads on the medial area of the knees.

- Type III: lipedema extends from the hips to the ankles.

- Type IV: arms and legs are affected.

- Type V: lipo-lymphedema occurs.

There is no known cure for lipedema. However, different applications such as Physiotherapy and Rehabilitation (PR) are recommended to improve the long-term prognosis of the disease. Correct diagnosis and treatment approaches help to prevent possible complications. Lipedema treatment is divided into conservative treatment (non-invasive) and invasive treatment. Invasive treatments such as liposuction become indicated when the disease is physically and psychologically difficult for the patient, or when there is no response to conservative treatment (11). One of the conservative treatments is PR applications. The aim of these applications is to control edema, reduce pain and hypersensitivity, increase the level of physical activity, and improve quality of life.

There is still a major deficiency in the diagnosis and treatment of lipedema and physicians, physiotherapists, and patients need more information and training (12). The goal of this review is to provide a better understanding of the effect and effectiveness of physiotherapy and rehabilitation treatment applications and to more clearly understand its value by coalescing existing studies.

\section{MATERIALS AND METHODS}

\section{Literature Review}

Published data in peer-reviewed articles evaluating the effects of PR in lipedema patients were analyzed. The present review is based upon bibliographic searches in PubMed (U.S. National Library of Medicine). Articles published in English up to June 2019 were identified using the following keywords: "lipedema" or "lipoedema" and "exercise in lipedema" or "physiotherapy and lipedema." Information obtained from bibliographic searches (title and topic of article, information in abstract) was used to determine whether to retain each article identified in this way. Subsequently data were analyzed by reading the entire text through full-text resources. To initiate the study, we collected information published about lipedema over the last 58 years (1951-2019).

The inclusion criteria were: (1) Studies examining the efficacy of physiotherapy in patients with lipedema; and (2) Studies regarding why lipedema patients may need physiotherapy. According to our results, 15 studies met inclusion criteria (Fig. 1; Table 1).

RESULTS

Complex Decongestive Physiotherapy 


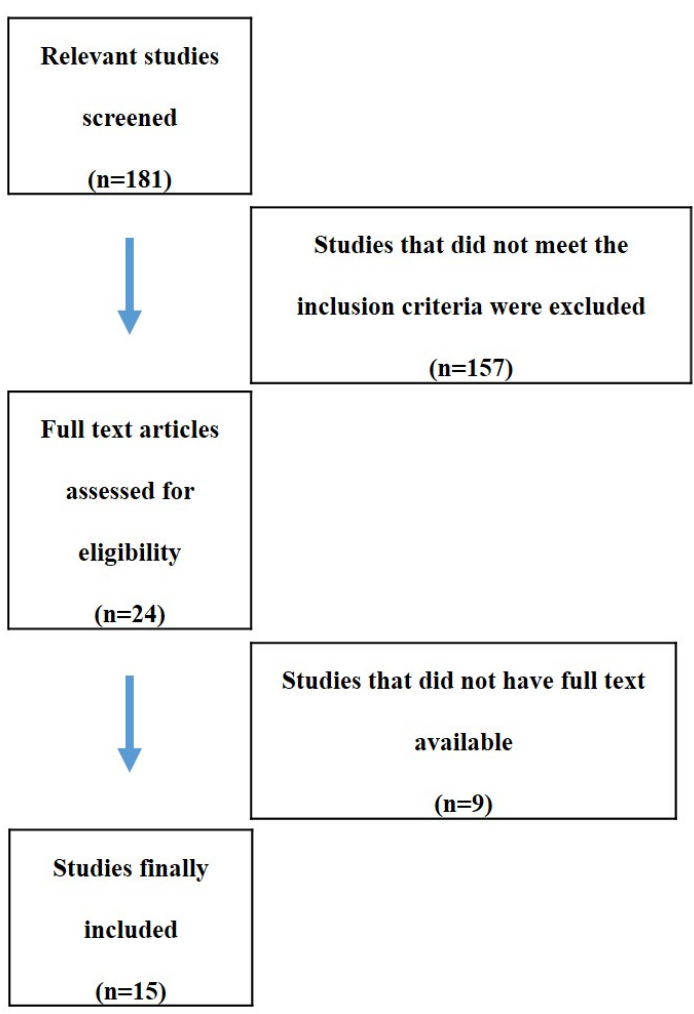

Fig. 1. Flowchart for selection of relevant studies included for analysis.

Complex Decongestive Physiotherapy (CDP) has four components of treatment for reducing and controlling edema: manual lymphatic drainage (MLD), compression application, physical exercise, skin care and self-management $(13,14)$.

There is a general consensus among experts that CDT is a safe and effective application when applied correctly $(15,16)$ The important effects of CDT consist of: (1) reduction of swelling; (2) increase of lymph drainage; (3) improvement of skin condition; (4) improvement of the functional activity; and (5) increase of quality of life (17).

CDP is considered the gold standard in the conservative treatment of lipedema (18). In patients with lipedema, the lymphatic system is thought to respond to MLD similarly to lymphedema. However, the response of subcutaneous adipose tissue to CDP is very slow. Therefore, compression bandage or garments may help reduce excess fat tissue (5).

Hematoma formations takes place even after minor trauma in lipedema patients. On the basis of clinical observations, CDP reduces the incidence of hematoma due to minor trauma rather than reducing leg volume. Hematomas may occur as a result of the change in capillary resistance and capillary fragility (CF). Only one study investigating the effects of MLD on CF in lipedema patients has been reported in the literature. In this study, CF was evaluated before and after CDP. 38 patients with lipedema participated in the study, 21 patients were treated with CDP and 17 patients were included in a control group. CDP consisted of daily MLD, intermittent pneumatic compression, and application of multi-layer short-stretch bandage. CF was evaluated with the vacuum suction method (VSM) using Parrot's angiosterrometer in both groups. No significant change in CF was observed in the control group after CDP but a significant decrease in CF was observed in the treatment group. This study suggests that CDP can significantly reduce CF in lipedema patients and may cause a decrease in hematoma formation (19).

Lipedema patients may have light pressureinduced or spontaneous pain. In a study performed by Szolnoky et al (20), they stated that CDP reduces pain perception beyond reducing leg volume. They evaluated the pain threshold of lipedema patients before and after treatment to investigate the effect of CDP on pain. The study was conducted in 38 patients with lipedema. Nineteen patients received CDP treatment while the control group received only moisturizing. CDP consisted of MLD once a day, intermittent pneumatic compression (IPC), and multilayered bandaging performed throughout a 5-day-course. Pain was evaluated with 3 different scales (10-item questionnaire, a pain rating scale (PRS), and the Wong-Baker Faces scale) before and after this treatment. Treatment resulted in a significant decrease in pain perception in all three scales. In the control group, only a significant decrease in 
TABLE 1

Characteristics of Included Studies

\begin{tabular}{|c|c|c|c|}
\hline Author, year & Sample size & Gender M/F & Mean Age (years) \\
\hline${ }^{2}$ Lester et al. 1940 & 119 & $1 / 118$ & NA \\
\hline${ }^{3}$ Herbst et al. 2015 & 51 & $1 / 50$ & $50 \pm 13$ \\
\hline${ }^{11}$ Schneider, 2018 & 30 & $\mathrm{~F}$ & 53.2 \\
\hline${ }^{18}$ Cordero et al. 2012 & $46 \mathrm{D}$ & Both & NR \\
\hline${ }^{19}$ Szolnoky et al. 2008 & 38 & F & $54.83(21 \mathrm{TG}) / 51.27(17 \mathrm{CON})$ \\
\hline${ }^{20}$ Szolnoky et al. 2011 & 38 & F & $53.89(19 \mathrm{TG}) / 55.7(19 \mathrm{CON})$ \\
\hline${ }^{21}$ Herbst, 2012 & NA & Both & NA \\
\hline${ }^{22}$ Witte et al. 2000 & NA & Both & NA \\
\hline${ }^{27}$ Chava et al. 2015 & 41 & $1 / 50$ & $50 \pm 13$ \\
\hline${ }^{29}$ De Wandele et al. 2013 & 78 & $8 / 70$ & $40.3 \pm 12.6$ \\
\hline${ }^{31}$ Reich-Schupke et al. 2017 & NA & Both & NA \\
\hline${ }^{37}$ Wienert, 2009 & NA & Both & NA \\
\hline${ }^{38}$ Stutz et al. 2011 & NA & NR & NA \\
\hline${ }^{41}$ Alwardat et al. 2019 & 712 & NR & NA \\
\hline${ }^{45}$ Szolnoky et al. 2008 & 23 & F & $51.3(13 \mathrm{CON}) / 50$ (10 TG) \\
\hline
\end{tabular}

Abbreviations: CON: Control Group; D: Documents; F: Female; M: Male; NA: Not Applicable; NR: Not Reported; TG: Treated Group

pain was seen in PRS. This study showed that CDP reduces leg volume and decreases pain perception in patients with lipedema (20).

\section{Exercise}

Lipedema is a chronic and progressive disease of adipose tissue characterized by abnormal subcutaneous fat deposition, leading to swelling especially of the lower limbs $(1,18)$. Unlike obesity, fat tissue is highly resistant to exercise in lipedema patients $(21,22)$. However, exercise is known to have an important effect on adipose tissue. Increase in adipose tissue in the body causes macrophage infiltration into adipose tissue leading to continuous low dose systemic inflammation $(23,24)$. This would suggest that there is a systemic inflammatory response in lipedema patients. Increasing IL-6 levels with moderate exercise can cause a decrease in the level of proinflammatory TNFalpha synthesized from adipocytes and therefore lead to an anti-inflammatory effect by increasing IL-10 and IL-1ra levels. IL-6 also stimulates fat oxidation by increasing lipolysis (25). In addition, exercise is thought to reduce edema in patients with lipedema (26). For these reasons, an appropriate exercise program should be added to the physiotherapy program for lipedema patients.

A study performed with 51 lipedema patients reported muscle weakness in more than $50 \%$ of patients in Stage 2 and 3 (27). This finding suggests that these patients could benefit from resistance exercises and therefore, resistance exercises should be added to their physiotherapy program. No study has examined the effects of resistance exercise training in lipedema patients. However, it is known that resistance exercise programs 
increase muscle strength in many patient groups (e.g., lymphedema) (28).

Hypermobility is not a condition reported in association with lipedema. However, in one study, $\sim 50 \%$ of patients with lipedema had a positive Beighton score ( $\geq 5)$ suggesting that they may have the common disorder known as Ehlers Danlos syndrome hypermobility type (EDS-HT) formerly type III) (29). Resistance exercises are used in hypermobility to induce strengthening and protection of the joints. Therefore, these exercises should be recommended as early as possible due to the potential risk of hypermobility (30).

Plantar arch abnormalities may be seen in lipedema patients (18). Abnormalities of the foot arch may cause postural deviations and shortness of muscles. Weakness and loss of flexibility may also occur in the muscles of the foot and lower extremities (31). Therefore, strength and flexibility of the muscles with foot arch abnormalities should be evaluated. Finally, resistance exercises and flexibility exercises should be added to the treatment program in accordance with these evaluation results.

Most lipedema patients experience increased swelling in warm weather, during airline travel, or during prolonged sitting (2). Therefore, these patients could be advised to avoid sitting for a long time, to perform simple exercises from their seat during a flight, and to wear compression garments before flight if appropriate.

Many lipedema patients have psychological problems such as depression and lack of self-confidence. In such cases, psychotherapy (32) and physiotherapy should be added. There was no study investigating the effects of exercise on self-esteem and depression in this patient group. However, other studies have shown that the appropriate dose of aerobic exercise is effective in the treatment of mild and moderate depression. (33) suggesting that exercise may be useful in the treatment of depression experienced by lipedema patients.

Women with stage 3 lipedema are also more likely to have obstructive sleep apnea (3).
Regular exercise may be beneficial for obstructive sleep apnea (34) in these patients as it increases ventilation and increases the strength of the respiratory muscles.

Pain is a common complaint in lipedema. Patients report spontaneous pain, pain during pressure and the feeling of tension, heaviness and burning (20). Regular moderate-intensity aerobic exercises are effective in reducing pain by increasing the production of endogenous opioids that are important in pain modulation. For this reason, moderate aerobic exercise could be added to the physiotherapy program of lipedema patients (35), and the importance of these exercises and their regular performance should be explained to patients.

Low levels of physical exercise and activity are a risk factor for further deterioration of lipedema, especially in combination with an increase in body weight. The main goal of therapeutic interventions is improving strength and fitness to enable an active lifestyle, which can help alleviate some symptoms, particularly in more moderate cases (36). Patients should be educated about the importance of a physiotherapy consisting of movements that focus on pumping the leg and calf muscles to increase lymphatic and venous flow and thereby prevent edema (37).

\section{Walk Training}

As lipedema progresses, increasing lower limb edema may lead to hip and knee joint damage resulting in orthopedic disorders and gait alterations and leading to impaired mobility (18).

Abnormal accumulation of fat in the legs and especially in the proximal hip results in a characteristic gait pattern (38). Lymphedema patients put their legs in an abduction position to minimize skin friction especially during the warmer months. As fat accumulation continues, the abduction angle in the hip increases and misaligned joint axes become clinically apparent. Abnormal stresses in the abduction position cause valgus deformity in the knee joint. Later, "skew- foot" deformity 
in the ankle joint and "varus shift" deformity in hip joint occur. The pseudo coxa-vara position caused by the abduction of the legs causes duck gait. Abnormal positions of the joints may also be seen even with normal weight. Knock knees deformity can be seen in lipedema patients and obese individuals. However, in contrast to the abnormal joint alignment caused by lipedema, knock knees deformity caused by obesity is due to valgus deformity of the hip joint (39).

When the literature was examined, no study investigating the effect of physiotherapy programs on gait pattern of lipedema patients was found. However, it is known that physiotherapy programs have positive effects on gait patterns in many patient groups $(40,41)$.

\section{Hydrotherapy}

There is no study investigating the effects of hydrotherapy of lipedema was found. In our opinion, hydrotherapy methods may be effective reducing the burden on the joints during exercise because patients with lipedema usually have a higher body mass index (BMI) (42). Lipedema may lead to degeneration of the joints even in the early stage of the disease. Also, as lipedema progresses, degeneration of the knee and hip joints may worsen (18). Exercises in water (especially swimming, water running, water aerobics) are seen as effective in reducing the strain on the joints and increasing lymph drainage in lipedema patients. Exercises against water resistance increase calorie utilization. However, weight training with water resistance alone is not effective in losing weight (43). To clarify the results, studies of hydrotherapy applications in lipedema patients should be conducted.

\section{Low-Frequency Vibrotherapy}

The lymphatic system can be stimulated via different routes. Specifically, there are devices that produce vibrational impulses and waves, which aim at altering cell functioning. In the physiotherapeutic practice, such devices have mainly been applied to treat pain but the body of evidence is inconclusive (44). We found a single study investigating the efficacy of vibration therapy in lipedema patients was found. In this study by Schneider et al, 30 lipedema patients were equally divided into 2 groups. The first group received only MLD while the second group received MLD with whole-body vibration therapy $(15 \mathrm{~Hz}-42 \mathrm{~Hz}$ ) for 6 sessions for $\mathbf{3 0}$ minutes. Results demonstrated a decrease in volume of the affected area and an increase in quality of life which was significantly higher in patients receiving MLD with vibration therapy compared to the group receiving MLD alone. (11). Vibration therapy might enhance the physiotherapy program in appropriate lipedema patients.

\section{Intermittent Pneumatic Compression}

Another approach used in the management of lipedema is IPC. Before IPC is applied, absolute and relative contraindications should be taken into account and risks considered. Adverse effects are extremely rare if IPC is used correctly (45). There are studies using IPC in lipedema patients $(19,20)$ but only one study investigating the effect of pneumatic compression alone was found. In this study, whether complete decongestive physiotherapy (CDP) alone or combined with IPC could improve the treatment outcome was investigated. Eleven patients received CDP (60 min) and thirteen patients received CDP (30 min) plus IPC (30 min) once a day, for 5 days in each group. CDP consisted of multilayered compression bandaging, exercise and skin care. Effects of the treatment were evaluated by limb volume reduction. Significant reduction in mean lower extremity volume was seen in both groups however no difference was seen between the groups in this small study (46).

\section{CONCLUSION}

This study is the first to review the effects of PR applications in patients with lipedema. Although the effects of PR for the treatment of 
lipedema are variable among studies, overall PR seems to be helpful. Regular exercise and active lifestyle may help prevent the development of lipedema-related complications. Physiotherapy should be used in combination with other treatment modalities. There is no study investigating the effects of PR before or after liposuction. In our opinion, PR can be valuable in every stage of lipedema management including before and after surgical procedures such as liposuction. The effectiveness of PR depends on the patients' stage and time of diagnosis. Even though it has positive effects, PR does not cure the disease. However, it can lead to positive effects via slowing the progression of the disease and preventing the development of complications. More and higher quality studies are needed to evaluate the effect and efficacy of PR in lipedema patients.

\section{Strengths and Limitations}

This is the first study to explore why patients with lipedema need physiotherapy in detail and examine in detail how physiotherapy is beneficial for this patient group. Light is also shed on future studies that are needed to expand knowledge on this topic. Limitations of the study are that the number of randomized controlled studies in literature is restricted and therefore the number of studies that could be included in this review was limited.

\section{CONFLICT OF INTEREST AND DISCLOSURE}

All authors declare no competing or potential financial conflicts of interest with respect to the research, authorship, and/or publication of this article.

\section{REFERENCES}

1. Fonder, MA, JW Loveless, GS Lazarus: Lipedema, a frequently unrecognized problem. J. Am. Acad. Dermatol. 57 (2007), S1-S3.

2. Allen, E, E Hines: Lipedema of the legs: A syndrome characterized by fat legs and orthostatic edema. Proc. Staff Meet. Mayo Clin, 15 (1940), 184-187.

3. Herbst, K, L Mirkovskaya, A Bharhagava, et al: Lipedema fat and signs and symptoms of illness, increase with advancing stage. Arch. Med. 7 (2015), 1-8.

4. Cornely, M: Lipoedema of arms and legs. Part 2. Phlebologie 40 (2011), 146-151.

5. Child, AH, KD Gordon, P Sharpe, et al: Lipedema: An inherited condition. Am. J. Med. Genet. Part A 152 (2010), 970-976.

6. Fife, CE, EA Maus, MJ Carter: Lipedema: A frequently misdiagnosed and misunderstood fatty deposition syndrome. Adv. Skin \& Wound Care 23 (2010), 81-92.

7. Mayes, J, G Watson: Direct effects of sex steroid hormones on adipose tissues and obesity. Obesity Rev. 5 (2004), 197-216.

8. Reich-Schupke, S, P Altmeyer, M Stücker: Thick legs-not always lipedema. JDDG: J. der Deutschen. Dermatol. Gesellschaft 11 (2013), 225-233.

9. Strößenreuther, R, R Baumeister: Lipödem und Cellulitis: Sowie andere Erkrankungen des Fettgewebes. Viavital-Verlag, 2001.

10. Schingale, F: Lymphoedema lipoedema. Schlütersche GmbH (2003), 20-21.

11. Schneider, R: Low-frequency vibrotherapy considerably improves the effectiveness of manual lymphatic drainage (MLD) in patients with lipedema: A two-armed, randomized, controlled pragmatic trial. Physiother. Theory Practice (2018), 1-8.

12. Reich-Schupke, S, E Mohren, M Stücker: Survey on the diagnostics and therapy of patients with lymphedema and lipedema. Der Hautarzt; Zeitschrift fur Dermatologie, Venerologie, und verwandte Gebiete 69 (2018), 471-477.

13. Lee, B, M Andrade, J Bergan, et al: Diagnosis and treatment of primary lymphedema. Consensus document of the International Union of Phlebology (IUP)-2009. Int. Angiol. 29 (2010), 454-470.

14. The Diagnosis and Treatment of Peripheral Lymphedema: 2020 Consensus Document of the International Society of Lymphology. Lymphology 53 (2020), 3-19.

15. Hinrichs, CS, JF Gibbs, D Driscoll, et al: The effectiveness of complete decongestive physiotherapy for the treatment of lymphedema following groin dissection for melanoma. J. Surg. Oncol. 85 (2004), 187-192.

16. Koul, R, T Dufan, C Russell, et al: Efficacy of complete decongestive therapy and manual lymphatic drainage on treatment-related lymphedema in breast cancer. Int. J. Radiat. Oncol. Biol. Phy. 67 (2007), 841-846.

17. Committee NMA: Position statement of the national lymphedema network. National Lymphedema Network: Berkeley, CA, USA, 2011.

18. Forner-Cordero, I, G Szolnoky, A Forner- 
Cordero, et al: Lipedema: An overview of its clinical manifestations, diagnosis and treatment of the disproportional fatty deposition syndrome-systematic review. Clin. Obesity 2 (2012), 86-95.

19. Szolnoky, G, N Nagy, R Kovács, et al: Complex decongestive physiotherapy decreases capillary fragility in lipedema. Lymphology 41 (2008), 161-166.

20. Szolnoky, G, E Varga, M Varga, et al: Lymphedema treatment decreases pain intensity in lipedema. Lymphology 44 (2011), 178-182.

21. Herbst, KL: Rare adipose disorders (RADs) masquerading as obesity. Acta Pharmacologica Sinica 33 (2012), 155.

22. Witte, CL, MH Witte, EC Unger, et al: Advances in imaging of lymph flow disorders. Radiographics 20 (2000), 1697-1719.

23. Yudkin, J: Inflammation, obesity, and the metabolic syndrome. Horm. Metab. Res. 39 (2007), 707-709.

24. Handschin, C, BM Spiegelman: The role of exercise and PGC1 $\alpha$ in inflammation and chronic disease. Nature 454 (2008), 463.

25. Fischer, CP: Interleukin-6 in acute exercise and training: What is the biological relevance? Exerc. Immunol. Rev. 12 (2006), 41.

26. Ratchford, EV, NS Evans: Approach to lower extremity edema. Curr. Treat. Options Cardio. Med. 19 (2017), 16.

27. Herbst, KL, L Mirkovskaya, A Bharhagava, et al: Lipedema fat and signs and symptoms of illness, increase with advancing stage. Arch. Med. 7 (2015), 10.

28. Nelson, NL: Breast cancer-related lymphedema and resistance exercise: A systematic review. J Strength Cond. Res. 30 (2016), 2656-2665.

29. De Wandele, I, L Rombaut, F Malfait, et al: Clinical heterogeneity in patients with the hypermobility type of Ehlers-Danlos syndrome. Res. Dev. Disabil. 34 (2013), 873-881.

30. Adam, M, H Ardinger, R Pagon, et al: Hypermobile Ehlers-Danlos Syndrome-GeneReviews ${ }^{\circledR}$. [Internet]. Seattle (WA): University of Washington, Seattle; 1993-2020. https://www.ncbi.nlm.nih.gov/books/NBK1279/

31. Kızılcı, H, F Erbahçeci: Evaluation of physical fitness in men with and without Pes Planus. Physioth. Rehabil. 27 (2016), 25-33.

32. Reich-Schupke, S, W Schmeller, WJ Brauer, et al: S1 guidelines: Lipedema. JDDG: J. Deutschen Dermatol. Gesellschaft 15 (2017), 758-767.

33. Dunn, AL, MH Trivedi, JB Kampert, et al: Exercise treatment for depression: efficacy and dose response. Am. J. Prevent. Med. 28 (2005), 1-8.
34. Norman, JF, SG Von Essen, RH Fuchs, et al: Exercise training effect on obstructive sleep apnea syndrome. Sleep Res. Online 3 (2000), 121-129.

35. Sluka, KA: Mechanisms and management of pain for the physical therapist. Lippincott Williams \& Wilkins, 2016.

36. Peled, AW, EA Kappos: Lipedema: Diagnostic and management challenges. Int. J. Womens Health 8 (2016), 389.

37. Okhovat, J-P, A Alavi: Lipedema: A review of the literature. Int. J. Low Extrem. Wounds 14 (2015), 262-267.

38. Wienert, V: DGP-Leitlinie Lipödem. Phlebologie 38 (2009), 164-167.

39. Stutz, J, S Wald: Liposuction in lipedema to prevent later joint complications. Vasomed 23 (2011), 6.

40. Patterson, SL, MM Rodgers, RF Macko, et al: Effect of treadmill exercise training on spatial and temporal gait parameters in subjects with chronic stroke: A preliminary report. J. Rehabil. Res. Dev. 45 (2008), 221.

41. Lord, SR, DG Lloyd, M Nirui, et al: The effect of exercise on gait patterns in older women: $\mathrm{A}$ randomized controlled trial. J. Gerontology Series A: Biol. Sci. Med. Sci. 51 (1996), M64-M70.

42. Alwardat, N, L Di Renzo, M Alwardat, et al: The effect of lipedema on health-related quality of life and psychological status: A narrative review of the literature. Eat Weight Disord. (2019), 1-6.

43. Weiner, R: Adipositas-Wann ist der Chirurg gefragt? DMW-Deutsche Medizinische Wochenschrift 140 (2015), 29-33.

44. Seco, J, FM Kovacs, G Urrutia: The efficacy, safety, effectiveness, and cost-effectiveness of ultrasound and shock wave therapies for low back pain: A systematic review. Spine J. 11 (2011), 966-977.

45. Schwahn-Schreiber, C, F Breu, E Rabe, et al: S1Leitlinie Intermittierende pneumatische Kompression (IPK, AIK). Der Hautarzt 69 (2018), 662-673.

46. Szolnoky, G, B Borsos, K Barsony, et al: Complete decongestive physiotherapy with and without pneumatic compression for treatment of lipedema: A pilot study. Lymphology 41 (2008), 40-44.
Murat Esmer, MSc
Department of Physiotherapy and Rehabilitation
Faculty of Health Sciences, Gazi University
Emniyet Neighborhood
Muammer Yasar Bostanci Street, Number: 16, Besevler
Ankara 06560, Turkey
Telephone number: +90531517 8500
E-mail: fztmrtesmer@hotmail.com 